\title{
BMJ Open Decisions to use antibiotics for upper respiratory tract infections across China: a large-scale cross-sectional survey among university students
}

\author{
Leesa Lin (1) , ${ }^{1,2}$ Elizabeth Fearon, ${ }^{1}$ Stephan Harbarth, ${ }^{3}$ Xiaomin Wang, ${ }^{4}$ \\ Chunling Lu, ${ }^{2}$ Xudong Zhou, ${ }^{4}$ James R Hargreaves ${ }^{1}$
}

To cite: Lin L, Fearon $\mathrm{E}$, Harbarth S, et al. Decisions to use antibiotics for upper respiratory tract infections across China: a large-scale cross-sectional survey among university students. BMJ Open 2020;10:e039332. doi:10.1136/ bmjopen-2020-039332

- Prepublication history for this paper is available online. To view these files, please visit the journal online (http://dx.doi org/10.1136/bmjopen-2020039332).

Received 14 April 2020 Revised 29 June 2020 Accepted 01 July 2020

Check for updates

(C) Author(s) (or their employer(s)) 2020. Re-use permitted under CC BY-NC. No commercial re-use. See rights and permissions. Published by BMJ.

${ }^{1}$ Public Health, Environments and Society, London School of Hygiene \& Tropical Medicine, London, UK

${ }^{2}$ Department of Global Health and Social Medicine, Harvard Medical School, Boston, Massachusetts, USA

${ }^{3}$ Infection Prevention and Control Division, University Hospitals Geneva, Geneve, Switzerland ${ }^{4}$ School of Medicine, Zhejiang University, Hangzhou, China

Correspondence to

Dr Xudong Zhou;

zhouxudong@zju.edu.cn

\section{ABSTRACT}

Objectives To investigate the decision-making process of Chinese university students with respect to antibiotic use for upper respiratory tract infections (URTIs).

Design A cross-sectional questionnaire study.

Setting The participants recruited from six universities across all Chinese regions from September to November 2015.

Participants A total of 2834 university students sampled across six Chinese regions who self-reported experiencing symptoms of URTI within the past month completed the survey.

Outcome measures The prevalence of decisions for treatment and antibiotic use for URTIs as well as knowledge about antibiotic use were measured by a selfadministrated questionnaire. Using regular and multinomial logistic regression a nd adapted health belief model, we identified and measured a number of variables as potential risk factors for antibiotic misuse behaviours in order to explain and predict people's treatment decisions and antibiotic use including knowledge, perceptions, access to antibiotics and cues to action.

Results Of the 2834 university students who selfdiagnosed a URTI, 947 (33.4\%) self-reported having taken antibiotics; among them, 462 (48.8\%) used nonprescription antibiotics, which came principally from left-over prescriptions (29.0\%) and over-the-counter purchases at retail pharmacies (67.3\%). One in four who sought care pressured their doctors for antibiotics; all received them. Those who perceived greater severity of the disease, had access to antibiotics, perceived benefits of using antibiotics (for the common cold: adjusted OR $(\mathrm{aOR})=2.55,95 \% \mathrm{Cl} 1.93$ to 3.38 or as anti-inflammatory drugs: $\mathrm{a} 0 \mathrm{R}=1.35,95 \% \mathrm{Cl} 1.12$ to 1.63 ), and were cued to action (eg, seeing presence of fever: $\mathrm{aOR}=2.05,95 \%$ Cl 1.62 to 2.60 or self-diagnosing their current infection as severe: $\mathrm{aOR}=1.86,95 \% \mathrm{Cl} 1.41$ to 2.45$)$, keeping antibiotics at home $(\mathrm{aOR}=2.27,95 \% \mathrm{Cl} 1.83$ to 2.81$)$ and access to over-the-counter antibiotics (aOR=2.00, 95\% Cl 1.63 to 2.45), were more likely to misuse antibiotics. Conclusions Misconceptions of antibiotic efficacy and easy access to antibiotics - with or without a prescription-were associated with antibiotic misuse among Chinese university students, which calls for context-appropriate multifaceted interventions in order to effectively reduce antibiotic misuse.
Strengths and limitations of this study

- Guided by the adapted Health Belief Model, this study identified risk factors influencing antibiotic use for upper respiratory tract infections (URTIs) in the Chinese context (eg, perceived efficacy of antibiotic use for URTIs and easy access to antibiotics), which can inform the design and development of community-based behavioural change interventions aiming to reduce antibiotic misuse.

- Heterogeneity exists in antibiotics-related knowledge and in its relationship with use.

- This study used a large sample size, with a response rate of $96 \%$, drawn from all six regions across China where respondents were evenly distributed across all demographic groups.

- Considering people may have multiple infections during the year and because our sampled population is generally younger, healthier and better educated than the general population, we anticipate antibiotic misuse among the Chinese general population to be more prevalent and severe than what has been presented here.

- The differences among the six provinces of different development levels from which the clustered samples of this study were drawn may be greater than those among individuals drawn from a random sample across the country-we have accounted for such variation at the province/university level in the analyses.

\section{INTRODUCTION}

Acute upper respiratory tract infections (URTIs), widely known as 'the common cold' and caused by several families of viruses, are the most common infectious diseases. ${ }^{1}$ The use of antibiotics is unnecessary for treating URTIs, as most URTIs are viral whereas antibiotics neither expedite recovery from infections nor prevent complications. ${ }^{2-4}$ Nevertheless, URTIs remain the most common reason for primary care seeking of antibiotics and antibiotic prescriptions in 
many countries, including China, which has one of the highest reported per capita rates of antibiotics use globally at $138 \mathrm{~g} /$ person. $^{5}$ Most Chinese antibiotic consumption occurs in outpatient settings, often unnecessarily for URTIs, which is a critical driver of inappropriate and excessive antibiotic use in China. ${ }^{6} 7$ Despite the magnitude of antibiotic misuse in China, there is little evidence towards understanding how people make URTI treatment decisions, and little effort has been made to apply health behaviour theories towards understanding such decision-making.

An individual's medical decisions, such as antibiotic use for URTIs, are influenced by their attitudes and perceptions of the illness and treatment options while these attitudes and perceptions themselves are heavily influenced by socioecological context. ${ }^{8-10}$ Adults may experience a URTI two to five times annually, ${ }^{1}$ making it so well recognised that, before consulting a physician, individuals commonly engage in self-diagnosis and decide a course of action-a majority of these self-recognised episodes were managed without seeking formal care. ${ }^{11}$ To date, a majority of studies on antibiotic use behaviours examine the issues from a traditional, knowledge-attitudes-practice perspective, assuming people would make more 'riskconscious' choices if informed of risks that could easily have been avoided (ie, improved knowledge and awareness leads to changes in practice). However, decision scientists argue that when people engage in healthcare decision-making, they focus on attaining a goal-curing of an illness. ${ }^{12}$ This thinking focuses on a positive rather than a negative outcome, where people accept the risks of drug resistance (for the immediate perceived benefits of antibiotic use) rather than intentionally taking risks. ${ }^{12}$

The 2019 British Medical Journal policy review of China's 10-year effort in health reform $^{13}$ clearly pointed to a current research and intervention gap that demonstrates there has been no improvement in primary care settings, where most of the population resides and the majority of antibiotic use takes place. Further, recent meta-analysis showed that despite being younger and better educated than the general public, university students' misuse of antibiotics has been a global health problem, especially in low-income and middle-income countries (LMIC). ${ }^{14}$ In China, university students represent the future opinion leaders and the next generation of parents, and therefore, could serve as an entry point for assessment and intervention to the future trajectory of antibiotic misuse in China. However, previous studies on Chinese university students were limited in scale to one school ${ }^{915}$ and/or one region ${ }^{915} 16$ and were not grounded in behavioural theories. In this study, we undertook a risk factor analysis for the public's antibiotic misuse behaviours for informing effective intervention development. Risk factors were identified using the Health Belief Model with an aim to explain and predict health behaviours with respect to antibiotic use for URTIs. Specifically, with selfdiagnosed URTI cases, we aimed to assess Chinese university students' (1) Antibiotic use. (2) Treatment decisions regarding care seeking and antibiotic use. (3) Prescription outcomes after seeking formal care, with a focus on antibiotic demands.

\section{METHODS}

\section{Population sample}

We used a cluster random sampling method to recruit university students across six Chinese regions for a crosssectional survey from September to November 2015. The single best comprehensive university ${ }^{17}$ in each of six sampled provinces in each region was identified as they represent educational elite, future opinion leaders and the next generation of parents of the country. Students attending these universities were eligible for enrolment. With assistance from school officials, class schedules were obtained the day prior to the survey. Classes were randomly selected from the timetable; every class had an equal chance of selection. All university students attending these classes were included. Over 95\% of students in the selected classes completed the questionnaire; some students did not participate due to lacking a phone or laptop at the time of survey. Pilot tests indicated the prevalence of URTIs among Chinese university students in the past month to be $25 \%-30 \%$. To ensure an adequate sample size for the planned subgroup analyses, we aimed to achieve a sample size per university of ca.1800 students. A total of 11192 students completed the survey. Among them, 25.32\% ( $\mathrm{n}=2834)$ self-reported experiencing symptoms of URTI ${ }^{1}$-including cold (cough, runny/stuffy nose), fever, sore throat, headache and influenza, either alone or in combinationwithin the past month. These respondents were evenly distributed across all demographic groups and included in this study. Further details on the survey's design and sampling methods have been previously described and published. ${ }^{10} 18$

\section{Data collection}

This study used a systematically developed questionnaire. Questions were tailored to the Chinese sociocultural context, as informed by literature review, ${ }^{8} 91516$ behavioural theories, and qualitative interviews with stakeholders and experts for face validity and content validity. A consent form was presented at the first section of the questionnaire and was signed by all participants, who were informed that participation was confidential, voluntary, could be terminated at any time, and that the questionnaire would take $\sim 5 \mathrm{~min}$ to complete. The survey was developed using Wen Juan Xing-a popular web-based platform for professional electronic questionnaires and data collection-and delivered via WeChat, China's most used communication application. The survey was finalised after a pilot test with 254 respondents to evaluate potential sources of response error and improve the instrument. The reliability and validity fit the requirements. 


\section{MODIFYING FACTORS}

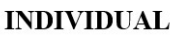

ACTION

SOCIO-CONTEXTUAL

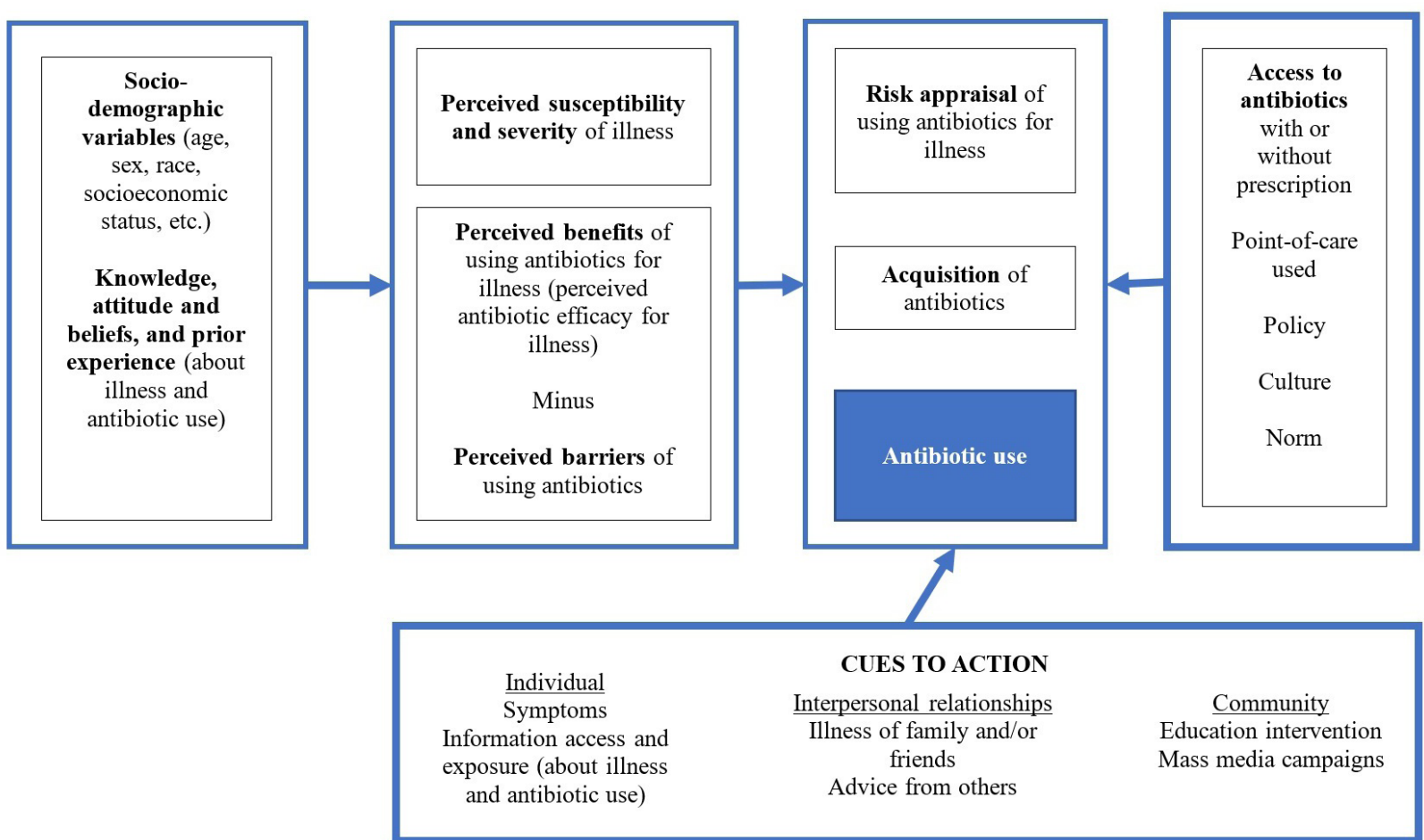

Figure 1 Modified health belief model for public antibiotic use.

\section{Behavioural frameworks}

We adapted the Health Belief Model ${ }^{19}{ }^{20}$ and the conceptual framework for analysis, as presented in figure 1. The study aims to understand the impact of individuals' perception of illness and treatment on one's decisions for antibiotic use while accounting for the complex interplay between factors at different levels of socioecological environment $^{21}$ (ie, individual, interpersonal and societal). Knowledge relates to antimicrobial resistance (AMR) awareness, ability to identify antibiotics and misconceptions. Perceptions involve expectations about the seriousness of the consequences of acquiring URTIs (ie, perceived severity) or the benefits of antibiotic treatment for URTI symptoms (perceived antibiotic efficacy). Cues to action are external determinants of health behaviours (eg, presence of symptoms).

\section{Outcome variables}

To assess the prevalence of antibiotic use for selfdiagnosed URTIs, participants were first assessed based on whether or not they had used antibiotics (with or without a prescription) for the symptoms they experienced. They were then categorised into three subgroups with respect to their treatment decisions for using antibiotics: no treatment or self-treated without antibiotics (reference group), self-treated with antibiotics, and sought formal care. Diagnostic outcomes of care seeking were categorised into three subgroups with respect to doctors' prescribing decisions for antibiotics: no antibiotic prescription (reference group), unnecessary antibiotic prescriptions for URTI symptoms and inappropriate antibiotic prescriptions due to patients' demands.

\section{Exposure variables}

Consulting the modified Health Belief Model for antibiotic use (figure 1), we included the following potential risk factors in our analyses:

1. Knowledge about antibiotics and resistance was measured by a series of factual statements related to AMR awareness (five items), ability to identify antibiotics (seven items) and misconception about the antibiotics as 'anti-inflammatory drugs' (one item).

2. Perceived severity of URTIs was measured by knowing that URTIs are self-limiting and will dissipate naturally.

3. Perceived antibiotic efficacy was measured by five factual statements about antibiotics' efficacy to treat URTIs.

4. Cues to action: (a) self-diagnosed severity of currentinfection, measured by numbers of cold symptoms experienced. (b) Presence of fever.

5. Access to antibiotics was measured by two yes/no questions: (a) Keeping antibiotics at home in the past year. (b) Over-the-counter purchase of non-prescription antibiotics in the past year.

Additionally, considering the Chinese socioecological environment regarding healthcare decisions, data on (6) Point of care used for care seeking was collected, ranging from hospitals above county level, county level hospitals, township hospitals and private clinics. 


\section{Covariates}

Sociodemographic characteristics were included as potential confounders for the association between each exposure and treatment decisions, including age, sex, household income, urbanicity, university major in medicine, having at least one parent with medical background and region/province.

\section{Statistical analysis}

First, we described and summarised participants' sociodemographic characteristics and risk factors by treatment decision/behavioural outcomes. Second, we developed a flow diagram to show pathways of different medical decision outcomes with respect to antibiotic use for URTIs. Third, we described responses to different domains of knowledge about antibiotic use and resistance. Fourth, to examine the association between each risk factor and outcome, we applied logistic regressions to estimate the OR (95\% CI) for 'treatment with antibiotic for URTIs' (vs 'no antibiotic use'). Last, we explored the associations with subgroups of antibiotic use for URTIs by self-medication and via formal care, and applied multinomial logistic regressions to estimate the relative risk ratio, RRR $(95 \%$ CI) for (1) 'self-treatment with antibiotics' and 'sought healthcare' (vs 'no treatment or self-medication without antibiotics'). For students who sought healthcare, we estimated RRR (95\% CI) for (2) 'receiving (unnecessary) prescriptions without request' and 'receiving (inappropriate) prescriptions due to patients request' (vs 'without an antibiotic prescription'). For each outcome and exposure, we first fitted an unadjusted model, and then a full model adjusted for potential confounders. These confounders-identified via published studies 891516 on public's antibiotic use-include: age, sex, household income, urbanicity, being a medical student or having at least one parent with a medical background, and hometown region of residence (university/province).

\section{Indirect patient and public involvement}

We did not directly include patient and public involvement in this study, but the database used was developed with stakeholders, including the general public who had previously experienced URTIs.

\section{Guidelines}

The guidelines for observational studies in epidemiology (Strengthening the Reporting of Observational studies in Epidemiology (STROBE) checklist) were followed during the preparation of the manuscript.

\section{RESULTS}

Distributions of decisions for treatment and antibiotic use for URTIS

As presented in figure 2, when the 2834 university students with self-diagnosed URTIs were asked about their choice of treatment, $20.4 \%$ decided against treatment $(n=579)$, $54.5 \%$ decided to self-treat (with or without antibiotics, $\mathrm{n}=1545)$, and a quarter sought formal care $(\mathrm{n}=710$, $25.1 \%$ ). A total of 947 students self-reported having taken antibiotics for URTI symptoms: $462(48.8 \%)$ used nonprescription antibiotics and the rest obtained a prescription. Non-prescription antibiotics came principally from left-over prescriptions $(29.0 \%)$ and over-the-counter purchases from pharmacies $(67.3 \%)$. One out of every four respondents who sought formal care admitted to pressuring their doctors for antibiotics $(\mathrm{n}=123,17.3 \%)$, with a $100 \%$ success rate.

\section{Knowledge and perceptions about antibiotic use and resistance}

Respondents were assessed on their knowledge about antibiotic use and resistance and perceptions on antibiotic efficacy and URTIs as a health threat. In table 1, the overall Cronbach's alpha, including all items, was 0.81 , with subscales of 0.71 (for AMR awareness) and 0.81 (for ability to identify antibiotics). Three quarters of participants $(74.2 \%-88.5 \%)$ reported being aware of the dangers posed by overuse of antibiotics. A majority of participants $(63.0 \%)$ had trouble distinguishing cold medicine (eg, Tylenol, Contac NT or Gankang) from antibiotics; $36.5 \%$ were unaware antibiotics were not antiinflammatory drugs; more than $60 \%$ had an incorrect perception of antibiotic efficacy for URTIs, either being unsure or wrongly stating that antibiotics might expedite recovery or alleviate symptoms.

\section{Factors associated with antibiotic use for URTIs}

Compared with those who did not use antibiotics for treatment of URTIs (see table 2), ability to identify antibiotics (adjusted OR (aOR) $=1.51,95 \%$ CI 1.17 to 1.94 ), perceived antibiotics to be effective for the common cold $(\mathrm{aOR}=2.55,95 \%$ CI 1.93 to 3.38$)$ or as anti-inflammatory drugs $(\mathrm{aOR}=1.35$, 95\% CI 1.12 to 1.63 ), not knowing that the common cold is self-limiting ( $\mathrm{aOR}=1.35,95 \%$ CI 1.12 to 1.62$)$, presence of fever ( $\mathrm{aOR}=2.05,95 \%$ CI 1.62 to 2.60), multiple symptoms experienced ( $\mathrm{aOR}=1.86,95 \% \mathrm{CI} 1.41$ to 2.45$)$ ), keeping antibiotics at home (aOR=2.27, 95\% CI 1.83 to 2.81 ) and access to over-the-counter antibiotics $(\mathrm{aOR}=2.00,95 \%$ CI 1.63 to $2.45)$ were associated with a higher likelihood of antibiotic use for URTIs.

\section{Factors associated with the treatment decisions for URTIs and} antibiotic use

Relative to those who did nothing or self-treated without antibiotics for URTIs (reference group in table 3), participants who self-medicated with antibiotics were more likely to have perceived antibiotic efficacy for URTIs (adjusted relative risk ratio (aRRR) $=3.03,95 \%$ CI 2.10 to 4.38 ), mistake antibiotics as anti-inflammatory drugs ( $\mathrm{aRRR}=1.40,95 \%$ CI 1.10 to 1.77), not know that the common cold is self-limiting (aRRR $=1.34,95 \%$ CI 1.05 to 1.71), experience multiple cold symptoms ( $\mathrm{aRRR}=1.96,95 \%$ CI 1.36 to 2.84), keep antibiotics at home $(\mathrm{aRRR}=4.68,95 \%$ CI 3.24 to 6.74$)$ and purchase over-the-counter antibiotics (aRRR=3.21, 95\% CI 2.34 to 4.41). Those who sought formal care were more likely to 


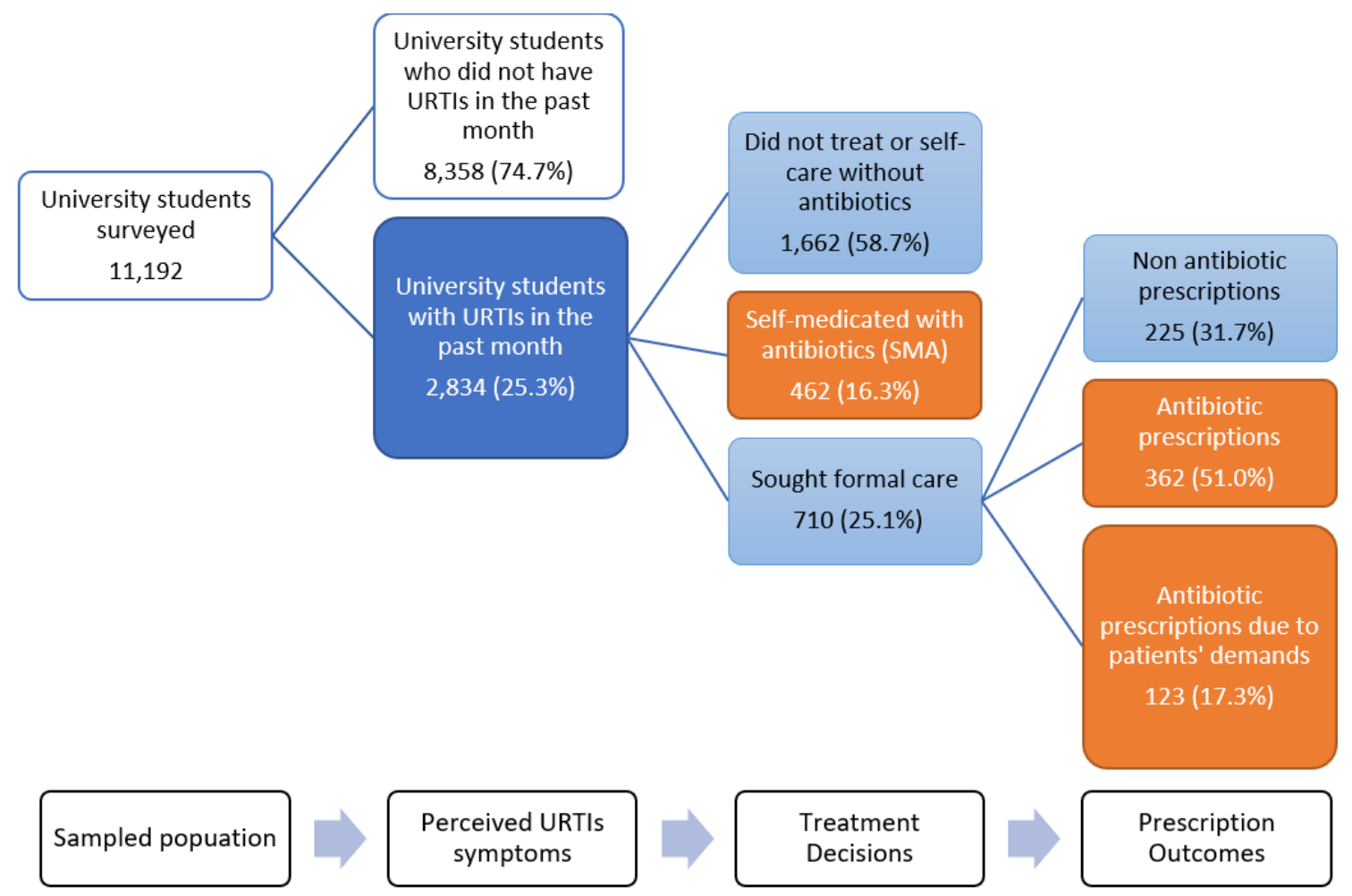

Estimate of contribution to antibiotic misuse for URTIs Demand side factors: $(462+123) /(462+485)=61.77 \%$ Supply side factors: $(485-123) /(462+485)=38.23 \%$

Figure 2 Medical decisions about antibiotic use for upper respiratory tract infections (URTIs) among Chinese university students.

have a high level of AMR awareness (aRRR $=0.61,95 \%$ CI 0.42 to 0.89 ), have not known that URTIs are self-limiting (aRRR $=1.66,95 \%$ CI 1.36 to 2.02), experienced multiple cold symptoms (aRRR $=1.64,95 \%$ CI 1.21 to 2.21 ) and had presence of fever ( $\mathrm{aRRR}=2.98,95 \%$ CI 2.32 to 3.83). Participants who had perceived antibiotics to be effective for the common cold (aRRR $=1.89,95 \%$ CI 1.38 to 2.57), kept antibiotics at home (aRRR $=1.24,95 \%$ CI 1.00 to 1.54$)$ and purchased over-thecounter antibiotics (aRRR=1.22, 95\% CI 0.99 to 1.51 ) were also more likely to seek formal care.

\section{Factors associated with antibiotic prescriptions for the treatment of URTIS}

Participants who sought care and had high levels of $A M R$ awareness had lower risks of demanding antibiotics. Relative to those who sought formal care but did not get an antibiotics prescription (reference group in table 4), participants who had high ability to identify antibiotics ( $\mathrm{aRRR}=6.35,95 \%$ CI 2.85 to 14.13), perceived antibiotics to be effective for the common cold ( $\mathrm{aRRR}=3.67$, 95\% CI 1.61 to 8.39 ) or as anti-inflammatory drugs (aRRR $=1.92,95 \%$ CI 1.11 to 3.33 ), presence of fever ( $\mathrm{aRRR}=3.24,95 \%$ CI 1.70 to 6.18), kept antibiotics at home ( $\mathrm{aRRR}=2.46,95 \%$ CI 1.33 to 4.56) and made over-the-counter purchase ( $\mathrm{aRRR}=3.69,95 \%$ CI 1.97 to 6.91 ) had a higher likelihood of demanding antibiotic prescriptions. Evidence of structural differences was observed in antibiotic prescribing outcomes in point of care, $54.3 \%$ of patients who sought care at tertiary hospitals and 52.7\% at township hospitals were prescribed antibiotics for URTIs, whereas $43.2 \%$ of county hospitals and $43.1 \%$ of local clinics gave antibiotic prescriptions for URTIs. All patients who demanded antibiotic prescriptions from a doctor received them.

\section{DISCUSSION}

We found that $33.4 \%$ of young Chinese educated in top universities $(\mathrm{n}=947)$ with URTIs used antibiotics; among them, $462(48.8 \%)$ used non-prescription antibiotics and additionally, a quarter of prescriptions originated from patients' demands $(\mathrm{n}=123,25.4 \%)$. We therefore estimated that the demand side is responsible for $61.8 \%$ of antibiotic use for URTIs $((462+123) / 947)$. Surprisingly, high ability to identify antibiotics was linked to higher likelihoods of antibiotic use, especially antibiotic prescriptions. Mistaking antibiotics as anti-inflammatory drugs, perceiving antibiotics as efficacious for treating URTIs and access to non-prescription antibiotics were strongly associated with antibiotic misuse, including selfmedication with antibiotics and demands for antibiotic prescriptions. We found non-prescription antibiotics are 
Table 1 Knowledge and perceptions about antibiotic use and resistance score distribution $(n=2834)$

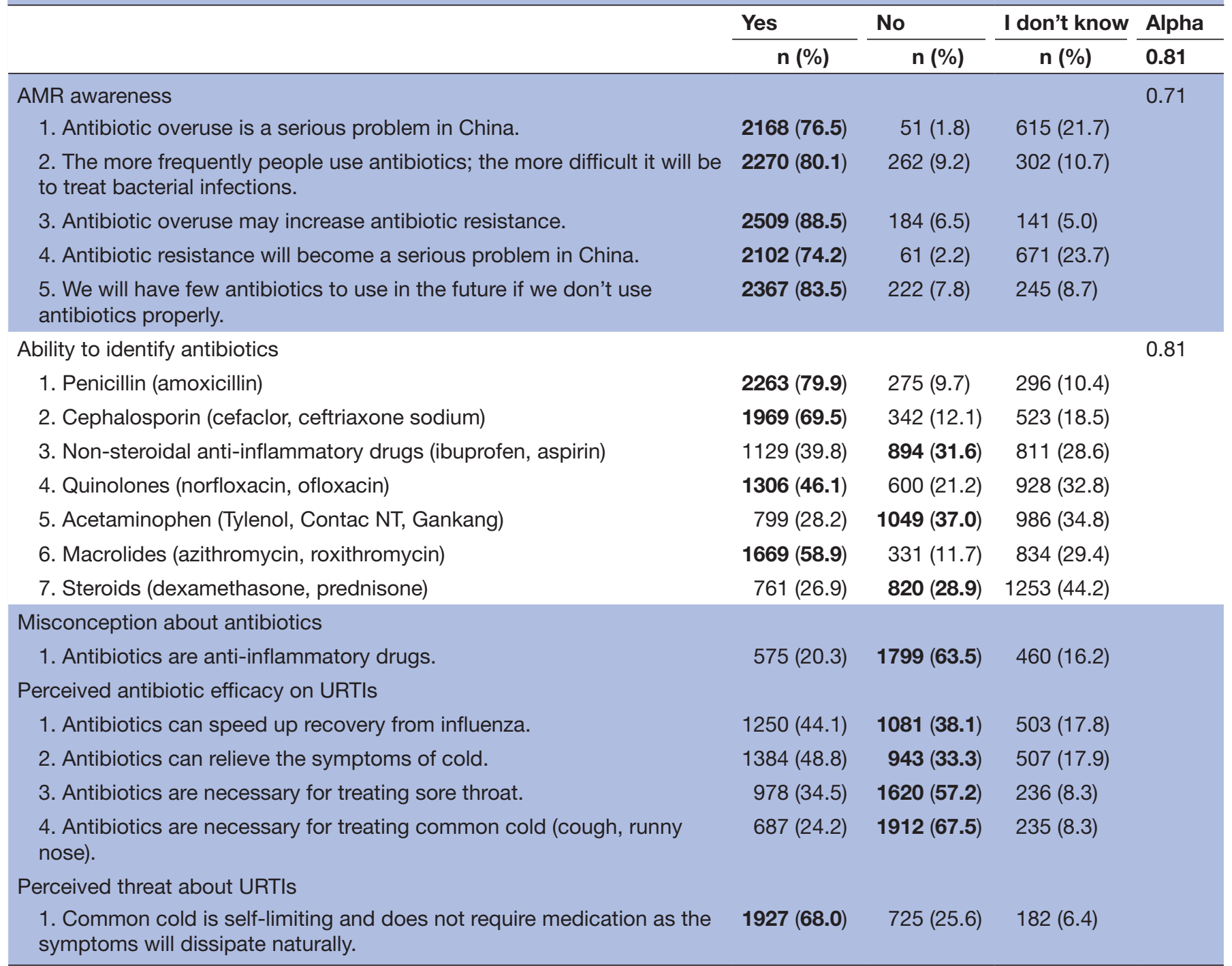

URTI, upper respiratory tract infection.

easily accessible in China: $68.2 \%$ of participants kept antibiotics at home at the time of the survey and $64.2 \%$ made over-the-counter purchases at least once within the past year. At health facilities, roughly $70 \%$ of clinicians prescribed antibiotics-most deemed as inappropriate and unnecessary-for URTI symptoms, and there was practically no barrier to accessing antibiotic prescriptions from a doctor.

\section{Strengths and limitations}

This study used a large sample size, with a response rate of $96 \%$, drawn from all six regions across China where respondents were evenly distributed across all demographic groups. As of today, the survey provided the most recent, nationwide data of its kind. This study is guided by the adapted Health Belief Model for analysis in explaining and predicting patients' treatment decisions and antibiotic use for URTIs. We found perceived infection severity, efficacy of antibiotic use for URTIs, barriers/access to antibiotics and cues to action are determinants of higher likelihoods of antibiotic use for URTIs, with or without prescriptions. In the Chinese context, our data further identified the demands of the health system-rather than supply-as the driving force for outpatient antibiotic use for URTIs, with a 1.6:1 ratio (62\% vs $38 \%$ ), and that access to antibiotics, with or without a prescription, was extremely easy. This model can guide the design and development of behavioural change interventions which aim to reduce antibiotic misuse in the community, with a focus on the complex interplay between individual/interpersonal/ societal factors and individuals' decisions on treatment and antibiotic use for URTIs. Compared with previous studies on similar populations in China ${ }^{915} 16$ and other low- and middle-income countries (LMIC), ${ }^{14}$ this study made a novel contribution to the field of AMR by rooting in a behavioural theory and conducting a holistic assessment on individual self-diagnostic and treatment decisionmaking processes with respect to antibiotics for URTIs, including the interaction between doctors and patients. 
Table 2 Logistic regression to assess factors associated with antibiotic use for upper respiratory tract infections (URTIs) among Chinese university students $(n=2834)$

\begin{tabular}{|c|c|c|c|c|c|}
\hline & \multirow{2}{*}{$\begin{array}{l}\text { All students with } \\
\text { URTIs ( } n=2834) \\
\mathrm{N}(\%)\end{array}$} & \multicolumn{3}{|c|}{ Antibiotic use for URTIs $(n=947,33.42 \%) \S$} & \multirow{2}{*}{$\begin{array}{l}P \\
\text { value*§}\end{array}$} \\
\hline & & $\mathrm{N}(\%)$ & OR (95\% Cl) & Adjusted OR (95\% Cl)‡ & \\
\hline \multicolumn{6}{|l|}{ Knowledge about antibiotics } \\
\hline \multicolumn{5}{|l|}{ AMR awareness } & \multirow[t]{4}{*}{0.97} \\
\hline Low & $181(6.39)$ & $73(7.71)$ & Reference & Reference & \\
\hline Medium & $571(20.15)$ & $210(22.18)$ & $0.86(0.61$ to 1.21$)$ & 1.02 (0.70 to 1.50$)$ & \\
\hline High & $2082(73.47)$ & $664(70.12)$ & 0.69 (0.51 to 0.95$)$ & 0.99 (0.70 to 1.42$)$ & \\
\hline \multicolumn{5}{|l|}{ Ability to identify antibiotics } & \multirow[t]{4}{*}{0.002} \\
\hline Low & $806(28.44)$ & $226(23.86)$ & Reference & Reference & \\
\hline Medium & $1267(44.71)$ & $470(49.63)$ & 1.51 (1.25 to 1.83$)$ & 1.37 (1.11 to 1.70$)$ & \\
\hline High & $761(26.85)$ & $251(26.50)$ & 1.26 (1.02 to 1.57$)$ & 1.51 (1.17 to 1.94$)$ & \\
\hline \multicolumn{6}{|c|}{ Perceived severity of the infection } \\
\hline \multicolumn{5}{|l|}{ Common cold is self-limiting } & \multirow[t]{3}{*}{0.002} \\
\hline Yes & $1927(68.00)$ & $581(61.35)$ & Reference & Reference & \\
\hline No/l don't know & $907(32.00)$ & $366(38.65)$ & 1.57 (1.33 to 1.85$)$ & 1.35 (1.12 to 1.62$)$ & \\
\hline \multicolumn{6}{|l|}{ Perceived antibiotic efficacy } \\
\hline \multicolumn{5}{|c|}{ Perceived antibiotic efficacy for URTIs } & \multirow[t]{4}{*}{$<0.0001$} \\
\hline No/low & $938(33.10)$ & $200(21.12)$ & Reference & Reference & \\
\hline Medium & $1476(52.08)$ & $542(57.23)$ & 2.14 (1.77 to 2.59 ) & 1.71 (1.39 to 2.10$)$ & \\
\hline High & $420(14.82)$ & $205(21.65)$ & 3.52 (2.75 to 4.50$)$ & 2.55 (1.93 to 3.38$)$ & \\
\hline \multicolumn{5}{|c|}{ Antibiotics are anti-inflammatory drugs } & \multirow[t]{3}{*}{0.001} \\
\hline No & $1799(63.48)$ & $524(55.33)$ & Reference & Reference & \\
\hline Yes/l don't know & 1035 (36.52) & $423(44.67)$ & 1.68 (1.43 to 1.97$)$ & 1.35 (1.12 to 1.63$)$ & \\
\hline
\end{tabular}

\section{Cues to action}

Severity of current infection (number of symptoms experienced) Low (1 symptom) $\quad 1488(52.51) \quad 395(41.71)$ Medium (two symptoms) $893(31.51) \quad 317(33.47)$

Reference $<0.0001$ High (three symptoms or $453(15.98)$ 235 (24.82)

1.52 (1.27 to 1.82 ) 1.37 (1.13 to 1.67 ) more)

2.98 (2.40 to 3.71 ) 1.86 (1.41 to 2.45 ) Fever $2235(78.86)$ 638 (67.37) Reference 309 (32.63) 2.67 (2.22 to 3.21 )

Reference $599(21.14)$ 2.05 (1.62 to 2.60$)$

Barriers/access to antibiotics

Keeping antibiotics at home

No

$900(31.76)$

$179(18.90)$

Reference

$<0.0001$

Yes

1934 (68.24)

$768(81.10)$

2.65 (2.20 to 3.20$)$

Reference

Over-the-counter purchase of non-prescription antibiotics in the past year
No
1015 (35.82)
$202(21.33)$
Reference
Yes
1819 (64.18)
$745(78.67)$
2.79 (2.33 to 3.34$)$
Reference

2.27 (1.83 to 2.81$)$

Sociodemographic characteristics

\begin{tabular}{|c|c|c|c|c|c|}
\hline Age & $21.13+(2.67)$ & & 1.02 (0.99 to 1.05$)$ & 1.00 (0.96 to 1.03$)$ & 0.79 \\
\hline Sex & & & & & 0.44 \\
\hline Male & $1476(52.08)$ & 496 (52.38) & Reference & Reference & \\
\hline Female & $1358(47.92)$ & $451(47.62)$ & 0.98 (0.84 to 1.15$)$ & $1.07(0.90$ to 1.28$)$ & \\
\hline
\end{tabular}

Continued 


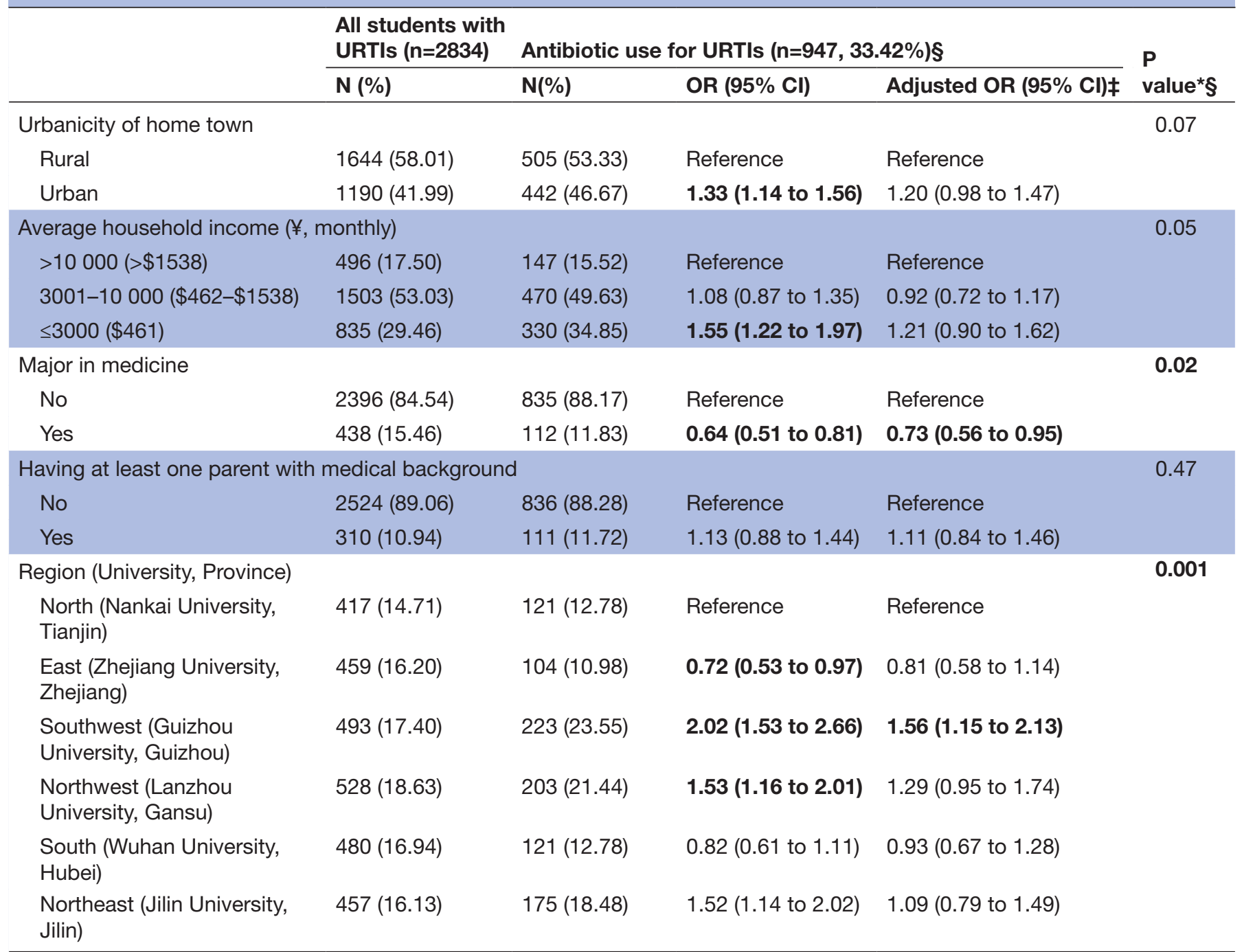

*Likelihood ratio tests for antibiotic use for URTIs.

†Mean (SD).

†Adjusted for age, sex, household income, urbanicity, university major in medicine, having at least one parent with medical background and region/province.

$\S B$ old values denote statistical significance at the $p<0.05$ level.

AMR, antimicrobial resistance.

Though the cross-sectional study design limited us from drawing causal relationships between knowledge and practice of antibiotic misuse, it helped to generate causal hypotheses and offered several points for intervention. Experiments, longitudinal studies or behavioural data are needed in the near future to avoid recall bias, an inherent limitation of self-reported survey data. Considering people may have multiple infections during the year and because our target population consisted of university students, who are generally younger, healthier and better educated than the general population, we anticipate antibiotic misuse among the Chinese general population to be more prevalent and severe than what has been presented here. Further, China is a vast and dynamic country; it is likely that some findings about this particular population may have changed over the last 5 years, which put constraints on the generalisability of the findings. Future studies should assess feasibility and appropriateness of the intervention proposed to the target population before full-scale implementation. As an example, a feasibility study conducted in rural Zhejiang in 2019 showed that findings from this study were applicable to its target population. ${ }^{22}$ Lastly, because the samples were clustered, the estimated standard errors used in significance tests may be biassed. In our case, samples were drawn from six universities across China, from provinces of different development levels; the differences among these provinces might be greater than those among individuals drawn from a random sample across the country. We have accounted for such variation at the province/university level in the analyses. 


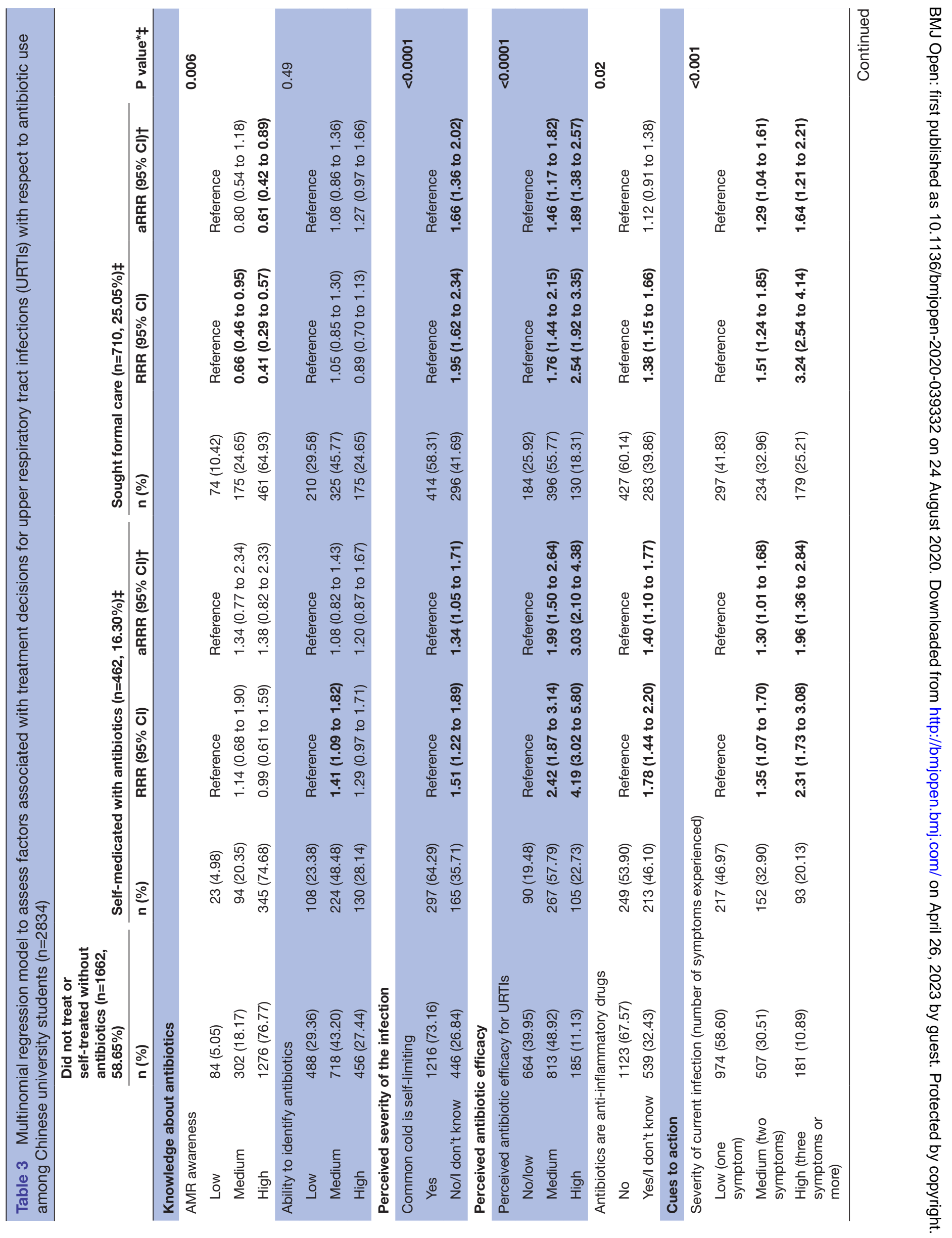




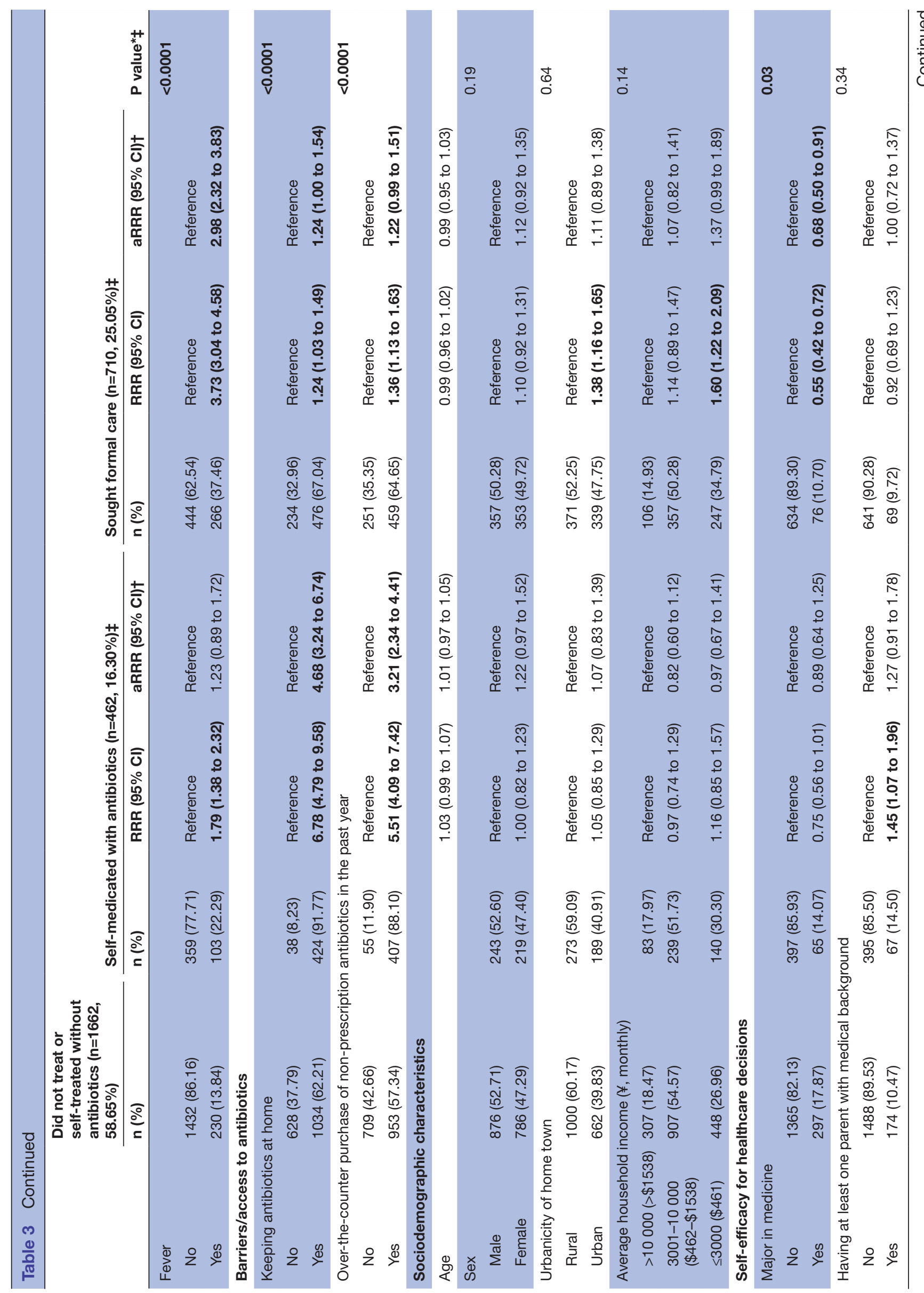




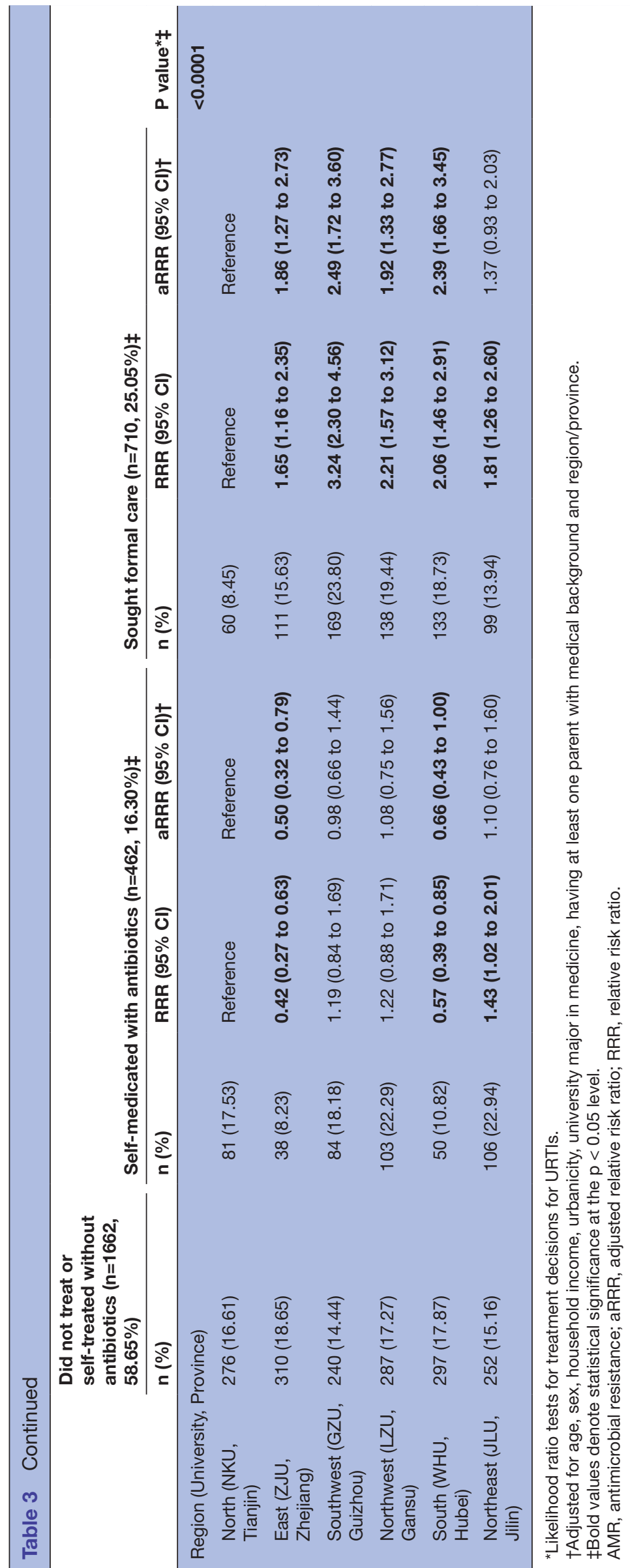

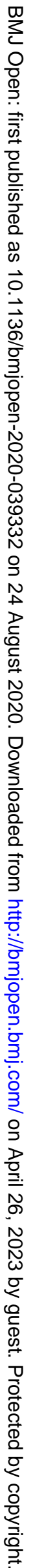




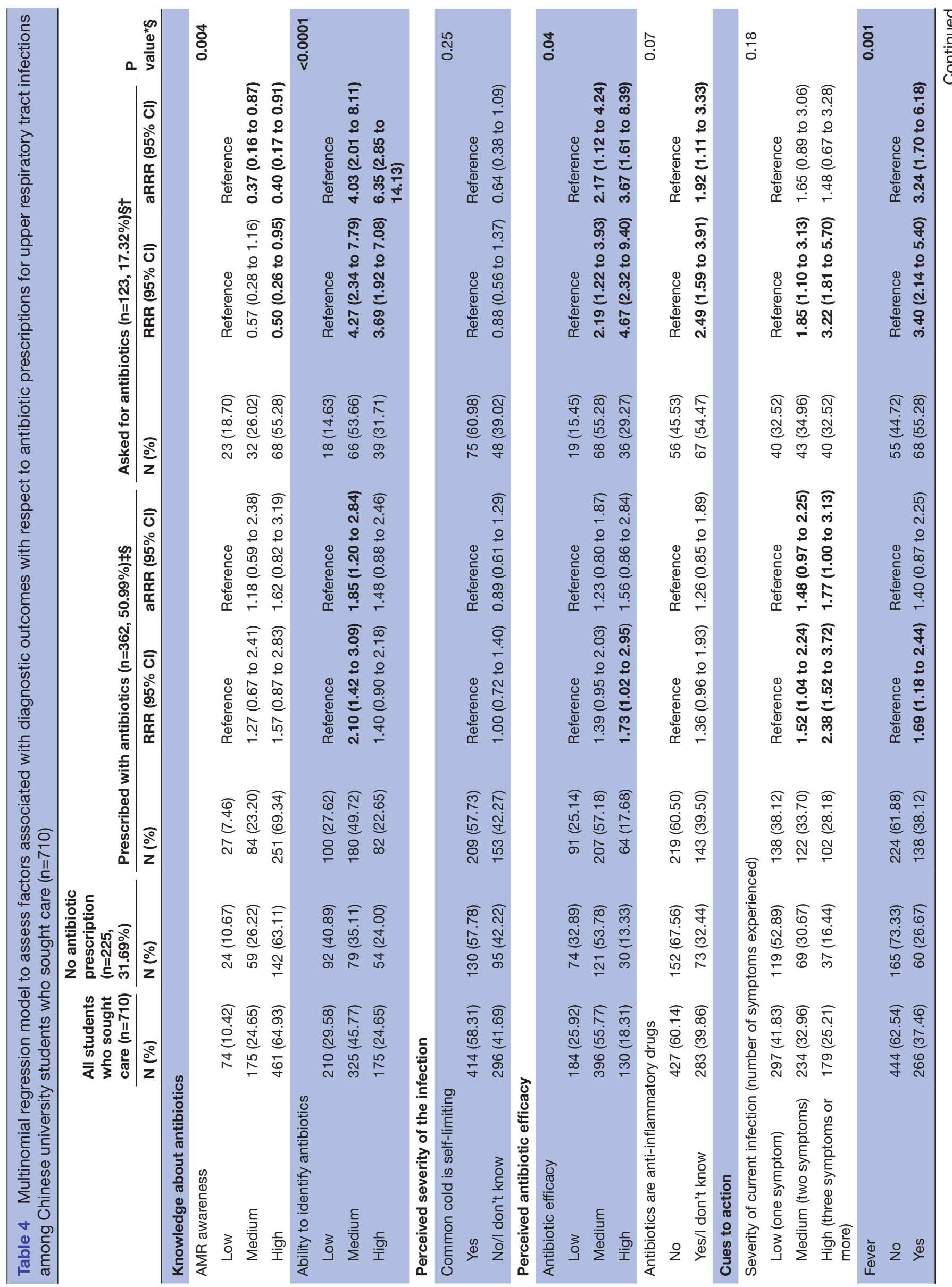




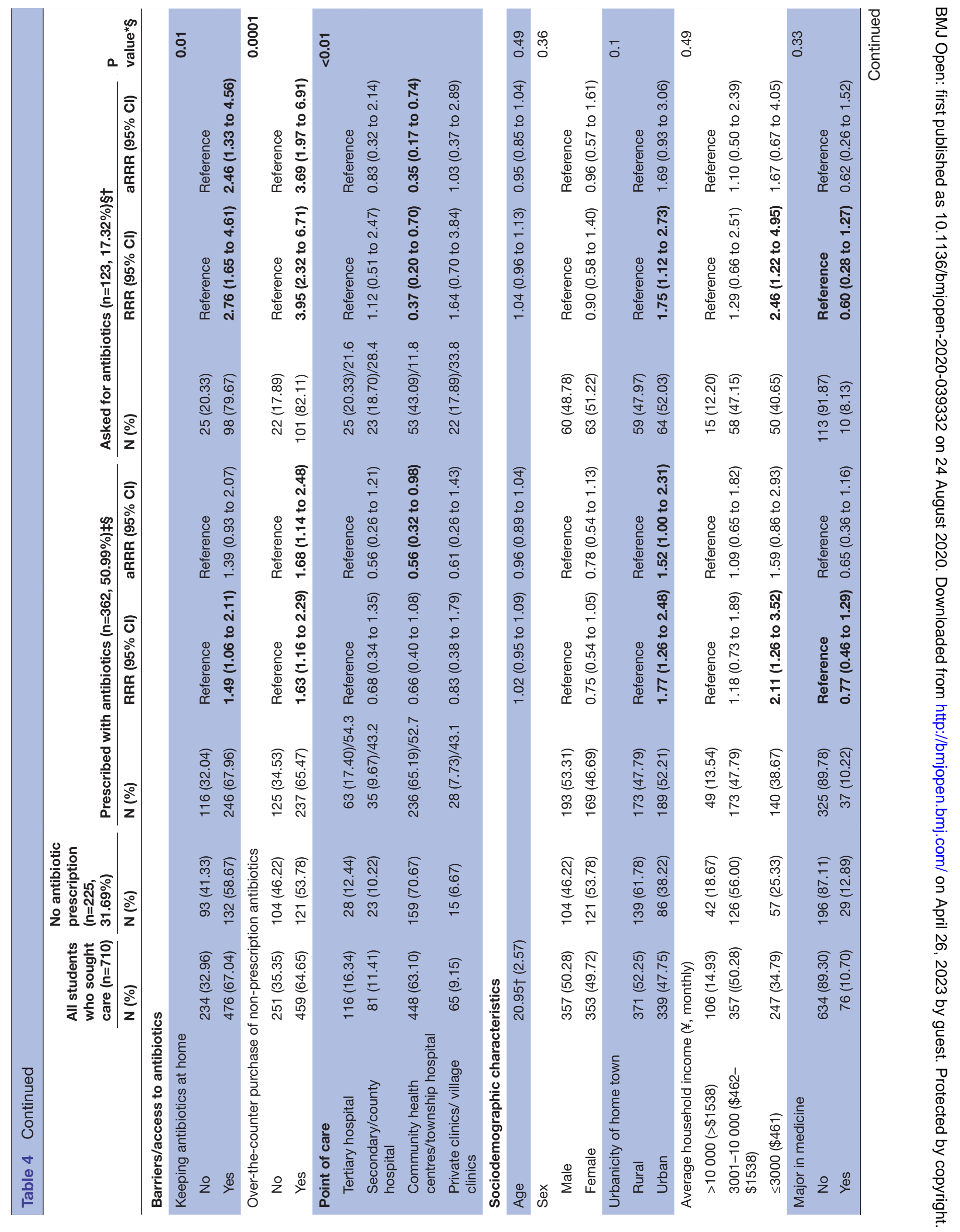




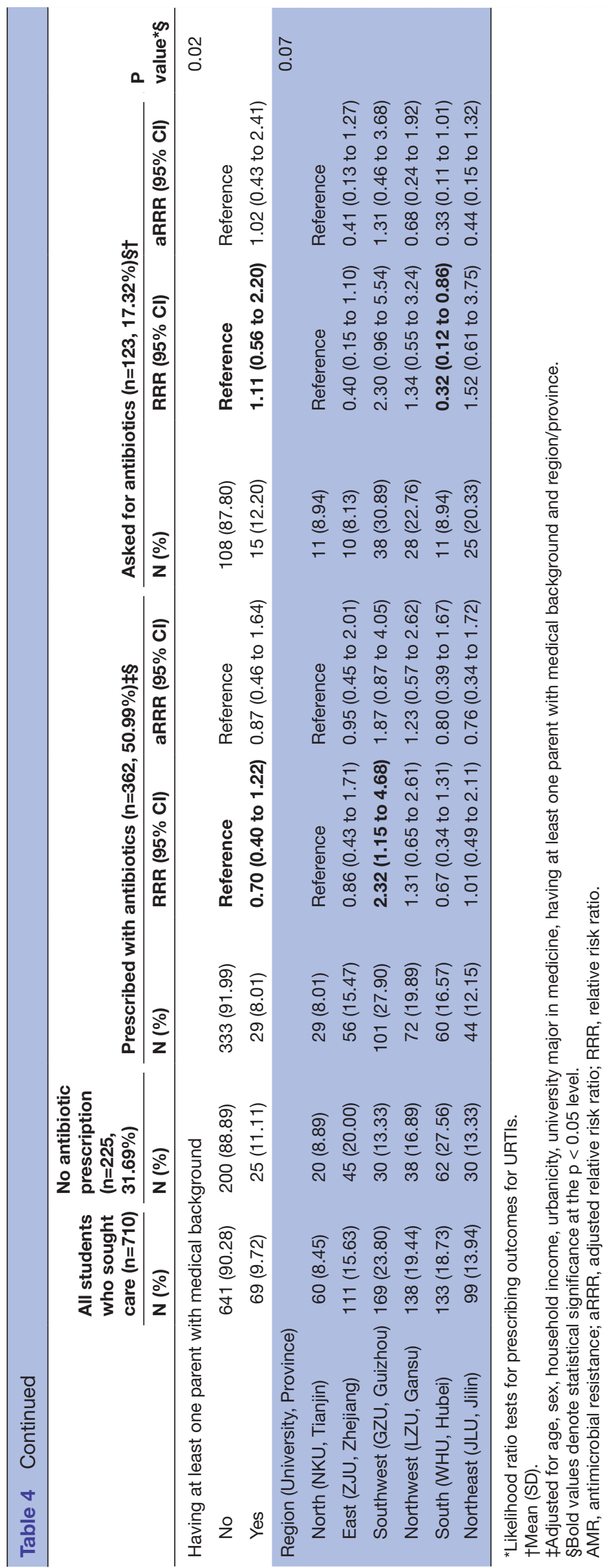

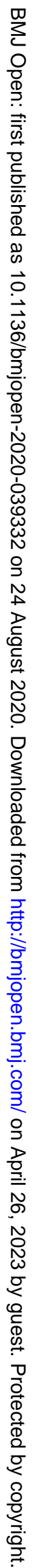




\section{Interpretation of findings}

First, we found that individual awareness of the danger of AMR was high among students, yet such awareness did not translate into prudent antibiotic use. This might imply the existence of an externality associated with antibiotic use for treating infections; despite a high awareness of AMR, the risks AMR imposes on others are unlikely to be felt directly or immediately by either the consumer or the supplier of treatment. Findings from this study identified a significant positive correlation between ability to identify antibiotics and self-medication, prescriptions, and demand for antibiotics. Previous studies demonstrated 'successful experiences' in the past for 'curing' a similar illness, and knowledge of the previously prescribed antibiotics could lead to improved ability to identify antibiotics and self-medicate with antibiotics (SMA), promoting antibiotic demand by patients, and leading to a cycle of overtreatment. ${ }^{15}$ 23-27 Our data suggest heterogeneity exists in the 'domain' of knowledge about antibiotics and its relationship with antibiotic practices for URTIs. Without sufficient knowledge about correct antibiotic efficacy, appropriate care for URTIs, and using antibiotics only under professional supervision, simply improving the public's ability to identify antibiotics alone could potentially cause greater misuse. Therefore, the common current practice $^{28} 29$ of grouping multiple aspects of antibiotics-related or AMR-related knowledge, attitudes and beliefs, and even practices into one score might not fully capture the complexity of their various associations with antibiotic use behaviours. A blanket awareness campaign or a simple intervention on clear labelling of antibiotics is likely to fail without adapting to the local context-a finding consistent with the recent assessment of the WHO awareness campaign. ${ }^{30}$ We found strong evidence showing that demanding antibiotic prescriptions was associated with household stockpiling and overthe-counter purchases of antibiotics. Interventions which target 'demanders' and 'heavy misusers' of antibiotics in the community and that correct the misperception of antibiotics' efficacy for URTIs or as anti-inflammatory drugs might reduce misuse.

In our data, all the participants who asked for antibiotics successfully received them. Even with good intentions, ${ }^{831-34}$ unrealistic patient expectations and pressure from patients or caregivers to prescribe antibiotics have been identified as major reasons why physicians prescribe antibiotics for self-limiting diseases. ${ }^{34-38}$ This indicates an urgent need for further training to help clinicians improve clinical skills and doctor-patient communication skills. Clinicians' overprescribing in China-incentivised by financial profits for health facilities ${ }^{39}$ - might have helped shape and reinforce common public misperceptions of antibiotics as effective for URTIs, which, in turn, further perpetuating misuse. This study showed $68.2 \%$ of participants stored antibiotics, which mainly came from over-the-counter purchases and previous prescriptions. The effect of keeping antibiotics at home on antibiotic (mis) use in the community is as profound as the impact of unnecessary prescriptions for URTIs through formal care. Since 2011, China has implemented policies (such as banning over-the-counter purchases and capping antibiotic prescriptions at $20 \%$ for county hospitals and above and $30 \%$ for township hospitals) to control antibiotic misuse, but the success of such initiatives has been limited due to poor enforcement. ${ }^{40-45}$ In our data, about $70 \%$ of patients with URTI who sought care were prescribed antibiotics; among them, over $50 \%$ of the patients received antibiotic prescriptions without prompting and close to $20 \%$ successfully obtained antibiotics through explicit requests. These data pointed to an urgent need for effective, context-tailored hospital stewardship programmes that improve adherence to clinical practice guidelines for antibiotic prescribing in China and enhance doctorpatient communication over antibiotic use. Consistent with other studies, ${ }^{9} 334647$ over-the-counter sales of nonprescription antibiotics at community pharmacies were found across China. Enforcing regulations regarding the sale of antibiotics, pack-based antibiotic dispensing systems, and public educational interventions to reduce consumer-driven prescriptions and left-over prescriptions could curb the main sources of antibiotics for selfmedication use.

\section{CONCLUSION}

Determinants of medical decisions for antibiotic use and care seeking are complex. It is critical to consider the heterogeneity of culture, health systems, and social norms in the assessment and intervention of decision-making regarding antibiotic use. This study provided urgently needed evidence for future interventional studies to address the challenges posed by the demand side and to improve the Chinese general population's antibiotic use.

Acknowledgements The authors thank the teachers and students involved in disseminating this survey and coordinating the study.

Contributors LL led the study design, conducted the literature search, created the tables and figures, conducted data analysis and interpretation, and drafted and revised the manuscript. EF, SH, XW, CL contributed to data interpretation, and commented on the initial and following revisions of the manuscript. XZ conceived the study, led data collection, contributed to data interpretation and commented on all drafts of this manuscript. JH supervised the data analysis and interpretation, and contributed significantly to the first draft and following revisions of the manuscript. All authors approved the final draft of this manuscript.

Funding The study was funded by the Zhejiang University Zijin Talent Programme.

Patient and public involvement The perspectives of the public were taken into account in designing the research question, but no patients and/or the public were directly involved in the development, conduct, reporting, or dissemination plans of the study. Refer to the Methods section for further details.

Patient consent for publication Not required.

Ethics approval The study protocol and survey were reviewed and approved by the Institutional Review Board at the School of Public Health Zhejiang University (No. ZGL20160922) and London School of Hygiene \& Tropical Medicine (No. 14678).

Provenance and peer review Not commissioned; externally peer reviewed.

Data availability statement The data sets generated and/or analysed during the current study are not publicly available due to the likelihood of compromising the privacy of participating individuals; the study materials are available from the corresponding author upon reasonable request. 
Open access This is an open access article distributed in accordance with the Creative Commons Attribution Non Commercial (CC BY-NC 4.0) license, which permits others to distribute, remix, adapt, build upon this work non-commercially, and license their derivative works on different terms, provided the original work is properly cited, appropriate credit is given, any changes made indicated, and the use is non-commercial. See: http://creativecommons.org/licenses/by-nc/4.0/.

ORCID iD

Leesa Lin http://orcid.org/0000-0003-4123-4762

\section{REFERENCES}

1 Eccles R. Understanding the symptoms of the common cold and influenza. Lancet Infect Dis 2005;5:718-25.

2 Alves Galvão MG, Rocha Crispino Santos MA, Alves da Cunha AJL. Antibiotics for preventing suppurative complications from undifferentiated acute respiratory infections in children under five years of age. Cochrane Database Syst Rev 2016;2:CD007880.

3 Kenealy T, Arroll B. Antibiotics for the common cold and acute purulent rhinitis. Cochrane Database Syst Rev 2013;6:CD000247.

$4 \mathrm{Kim}$ NN, Marikar D. Antibiotic prescribing for upper respiratory tract infections: NICE guidelines. Arch Dis Child Educ Pract Ed 2020;105:104-6.

5 Qiao M, Ying G-G, Singer AC, et al. Review of antibiotic resistance in China and its environment. Environ Int 2018;110:160-72.

6 Zhang Z, Hu Y, Zou G, et al. Antibiotic prescribing for upper respiratory infections among children in rural China: a crosssectional study of outpatient prescriptions. Glob Health Action 2017;10:1287334.

7 Li J, Song X, Yang T, et al. A systematic review of antibiotic prescription associated with upper respiratory tract infections in China. Medicine 2016;95:e3587

8 Yu M, Zhao G, Stålsby Lundborg C, et al. Knowledge, attitudes, and practices of parents in rural China on the use of antibiotics in children: a cross-sectional study. BMC Infect Dis 2014;14:112.

9 Lv B, Zhou Z, Xu G, et al. Knowledge, attitudes and practices concerning self-medication with antibiotics among university students in Western China. Trop Med Int Health 2014;19:769-79.

10 Wang X, Peng D, Wang W, et al. Massive misuse of antibiotics by university students in all regions of China: implications for national policy. Int J Antimicrob Agents 2017:50:441-6.

11 Kleinman A, Eisenberg L, Good B. Culture, illness, and care: clinica lessons from anthropologic and cross-cultural research. Ann Intern Med 1978;88:251-8.

12 Brun W, Teigen HK. Anticipating the future: appraising risk and uncertainty. In: Ray Crozier RR, Svenson O, eds. Decision making: cognitive models and explanations. 1st edn. London, New York: Routledge, 1997.

13 He P, Sun Q, Shi L, et al. Rational use of antibiotics in the context of China's health system reform. BMJ 2019;365:14016.

14 Xu R, Mu T, Wang G, et al. Self-medication with antibiotics among university students in LMIC: a systematic review and meta-analysis. J Infect Dev Ctries 2019;13:678-89.

15 Pan H, Cui B, Zhang D, et al. Prior knowledge, older age, and higher allowance are risk factors for self-medication with antibiotics among university students in southern China. PLoS One 2012;7:e41314.

16 Huang Y, Gu J, Zhang M, et al. Knowledge, attitude and practice of antibiotics: a questionnaire study among 2500 Chinese students. BMC Med Educ 2013;13:163.

17 ShanghaiRanking Consultancy. Best Chinese universities ranking overall ranking - 2015, 2015. Available: http://www.shanghairanking. com/chinese_universities_rankings/overall-ranking-2015.html [Accessed Jun 2019].

$18 \mathrm{Hu}$ Y, Wang X, Tucker JD, et al. Knowledge, attitude, and practice with respect to antibiotic use among Chinese medical students: a multicentre cross-sectional study. Int J Environ Res Public Health 2018;15:1165.

19 Ancillotti M, Eriksson S, Veldwijk J, et al. Public awareness and individual responsibility needed for judicious use of antibiotics: a qualitative study of public beliefs and perceptions. BMC Public Health 2018;18:1153.

20 Janz NK, Becker MH. The health belief model: a decade later. Health Educ Q 1984;11:1-47.

21 Weissman J, Besser RE. Promoting appropriate antibiotic use for pediatric patients: a social ecological framework. Semin Pediatr Infect Dis 2004;15:41-51.
22 Lin L, Wang X, Wang W, et al. Cleaning up China's medical cabinetan antibiotic take-back programme to reduce household antibiotic storage for unsupervised use in rural China: a mixed-methods feasibility study. Antibiotics 2020;9:212.

23 Reynolds L, McKee M. Factors influencing antibiotic prescribing in China: an exploratory analysis. Health Policy 2009;90:32-6.

24 Ocan M, Obuku EA, Bwanga F, et al. Household antimicrobial selfmedication: a systematic review and meta-analysis of the burden, risk factors and outcomes in developing countries. BMC Public Health 2015;15:742.

25 Núñez M, Tresierra-Ayala M, Gil-Olivares F. Antibiotic self-medication in university students from Trujillo, Peru. Medicina Universitaria 2016;18:205-9.

26 Alhomoud F, Aljamea Z, Almahasnah R, et al. Self-medication and self-prescription with antibiotics in the middle East-do they really happen? A systematic review of the prevalence, possible reasons, and outcomes. Int J Infect Dis 2017;57:3-12.

27 Nepal G, Bhatta S. Self-medication with antibiotics in who Southeast Asian region: a systematic review. Cureus 2018;10:e2428-e.

28 Chai J, Coope C, Cheng J, et al. Cross-sectional study of the use of antimicrobials following common infections by rural residents in Anhui, China. BMJ Open 2019;9:e024856.

29 Huh K, Chung DR, Kim SH, et al. Factors affecting the public awareness and behavior on antibiotic use. Eur J Clin Microbiol Infect Dis 2018;37:1547-52.

30 Huttner B, Saam M, Moja L, et al. How to improve antibiotic awareness campaigns: findings of a WHO global survey. BMJ Glob Health 2019;4:e001239.

31 Ding L, Sun Q, Sun W, et al. Antibiotic use in rural China: a cross-sectional survey of knowledge, attitudes and self-reported practices among caregivers in Shandong Province. BMC Infect Dis 2015;15:576.

32 Sun Q, Dyar OJ, Zhao L, et al. Overuse of antibiotics for the common cold - attitudes and behaviors among doctors in rural areas of Shandong Province, China. BMC Pharmacol Toxicol 2015;16:6.

33 Quan-Cheng K, Jian-Guo W, Xiang-Hua L, et al. Inappropriate use of antibiotics in children in China. Lancet 2016;387:1273-4.

34 Zhang Z, Zhan X, Zhou H, et al. Antibiotic prescribing of village doctors for children under 15 years with upper respiratory tract infections in rural China: a qualitative study. Medicine 2016;95:e3803.

35 Branthwaite A, Pechère JC. Pan-European survey of patients attitudes to antibiotics and antibiotic use. J Int Med Res 1996;24:229-38.

36 Harbarth S, Albrich W, Brun-Buisson C. Outpatient antibiotic use and prevalence of antibiotic-resistant pneumococci in France and Germany: a sociocultural perspective. Emerg Infect Dis 2002;8:1460-7.

37 McCullough AR, Parekh S, Rathbone J, et al. A systematic review of the public's knowledge and beliefs about antibiotic resistance. $J$ Antimicrob Chemother 2016;71:27-33.

38 Fletcher-Lartey S, Yee M, Gaarslev C, et al. Why do general practitioners prescribe antibiotics for upper respiratory tract infections to meet patient expectations: a mixed methods study. BMJ Open 2016;6:e012244.

$39 \mathrm{Li} \mathrm{Y}$. China's misuse of antibiotics should be curbed. BMJ 2014;348:g1083.

40 Yip WC-M, Hsiao W, Meng Q, et al. Realignment of incentives for health-care providers in China. Lancet 2010;375:1120-30.

41 Heddini A, Cars O, Qiang S, et al. Antibiotic resistance in China--a major future challenge. Lancet 2009;373:30.

$42 \mathrm{Li} \mathrm{Y}$. China's misuse of antibiotics should be curbed. BMJ 2014;348:g1083.

43 Wang Z, Zhang H, Han J, et al. Deadly SINS of antibiotic abuse in China. Infect Control Hosp Epidemiol 2017;38:758-9.

44 Xiao Y, Li L. Legislation of clinical antibiotic use in China. Lancet Infect Dis 2013;13:189-91.

45 Wang L, Zhang X, Liang X, et al. Addressing antimicrobial resistance in China: policy implementation in a complex context. Global Health 2016;12:30

46 You JHS, Yau B, Choi KC, et al. Public knowledge, attitudes and behavior on antibiotic use: a telephone survey in Hong Kong. Infection 2008;36:153-7.

47 Wun YT, Lam TP, Lam KF, et al. The public's perspectives on antibiotic resistance and abuse among Chinese in Hong Kong. Pharmacoepidemiol Drug Saf 2013;22:241-9. 